\title{
Two-beam gyrotron with frequency multiplication
}

\author{
I. V Bandurkin $^{1}$, M. Yu. Glyavin ${ }^{1}$, T. Idehara ${ }^{2}$, and A. V. Savilov ${ }^{1}$ \\ ${ }^{1}$ Institute of Applied Physics, Russian Academy of Sciences, Nizhny Novgorod, Russia, iluy@appl.sci-nnov.ru \\ ${ }^{2}$ Center for Development of Far-Infrared Region, University of Fukui, Fukui, Japan
}

\section{Introduction}

A well-known method of weakening the requirements for the magnitude of the operating magnetic field in gyrotrons is generation at high cyclotron harmonics. Gyrotrons have long demonstrated the ability to work at high cyclotron harmonics at frequencies above $1 \mathrm{THz}$. Nevertheless, the majority of gyrotrons using the conventional magnetron-injector gun (MIG) operate at a cyclotron harmonic not higher than the second. First of all, this is due to the aggravation of the selectivity problem, since a hollow electron beam produced in such a gun is coupled to many modes of the system. Parasitic oscillations at low cyclotron harmonics, as a rule, have higher coupling coefficients and therefore get an advantage in excitation. Partly, the problem of selectivity can be solved by the use of an axis-encircling electron beam, since the spectrum of the modes interacting with it is relatively rare. Nevertheless, the electron gun capable of formation of such a beam is rather complicated in comparison with the MIG, and the obtained electron current is limited due to the small area of the beam cross section. An alternative method of increasing the selectivity can be the use of the frequency multiplication effect, when the gyrotron is excited at the fundamental cyclotron resonance and simultaneously emits at one of the higher harmonics of the fundamental frequency. We propose a variant of such a gyromultiplier scheme, which can provide a relatively high generation efficiency in the terahertz range due to the use of two electron beams.

\section{Two-beam gyromultiplier scheme}

The main problem in the realization of a singlebeam self-excited gyromultiplier is the fast saturation along the system of high-frequency currents at all cyclotron harmonics (Fig. 1a) [1-4]. In a two-cavity scheme, this leads to the operation of a second cavity at an overbunched beam with a low efficiency. A single-cavity scheme requires the use of special methods for providing a double resonance (at low and high cyclotron harmonics) in the same electrodynamic volume, which greatly complicates the system.

We suggest a two-cavity scheme in which the electron overbunching problem is solved by using two electron beams (Fig. 1b). One of these beams is strongly coupled with the mode of the first cavity at the fundamental frequency and ensures its selfexcitation. The second beam has a relatively weak coupling with this low-frequency mode due to the correct choice of its radius. Therefore, its modulation in the first cavity is also weak, and the maximum bunching in it is achieved only in the second cavity, where, at this time, it is strongly coupled with the mode at a high harmonic (Fig 1c).

a)

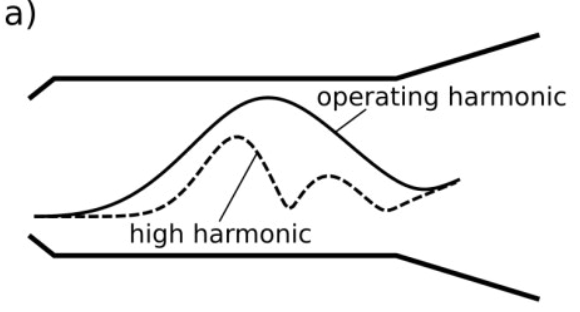

\section{b)}

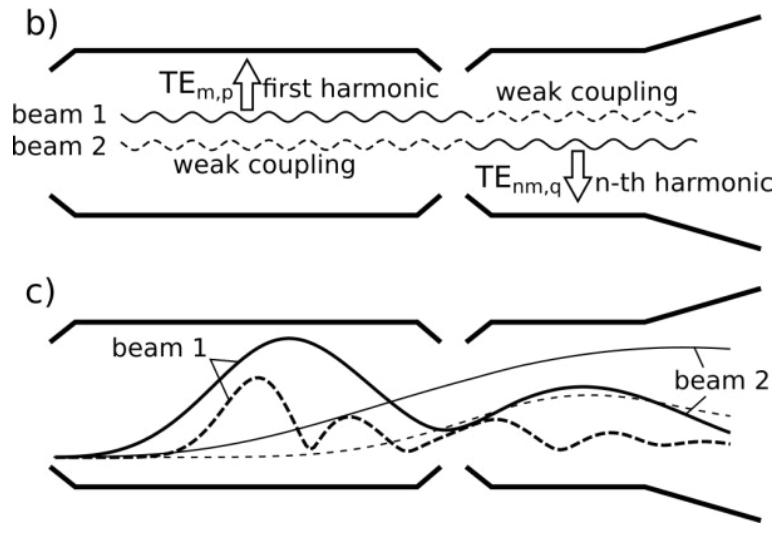

Fig. 1. Typical distributions of high-frequency current at various harmonics of the operating frequency in a conventional gyrotron (a), proposed two-cavity two-beam gyromultiplier scheme (b) and distribution of high-frequency currents in it (c).

\section{Selection of operating modes and beams radii}

For an example design we have chosen operation at first and third cyclotron harmonics, at the frequencies close to $400 \mathrm{GHz}$ and $1.2 \mathrm{THz}$, correspondingly. A characteristic feature of gyromultipliers is that the azimuthal indices of operating modes should be multiples of the same coefficient as their frequencies and cyclotron harmonic numbers. On the one hand, this additionally increases the selectivity of the harmonic generation, but, on the other hand, this requirement complicates the selection of operating modes. It is simplest to choose the system parameters in the case of axisymmetric modes with a zero azimuthal index. For instance, when a $\mathrm{TE}_{0,4}$ and $\mathrm{TE}_{0,13}$ modes are selected at the first and third cyclotron harmonics, the beam radii can be chosen so that each of the beams is well coupled with its mode, and simultaneously is near the zero of excitation of the other mode (Fig. 2a). Moreover, since the starting current at the fundamental harmonic is low, it is reasonable to put the larger part of the total electron beam current to the outer beam destined for the high harmonic operation. In the discussed design, the ratio of electron currents in inner and outer beams equals to 1:9. Nevertheless, when choosing the second beam radius equal to $0.44 \mathrm{~mm}$ in 
order to get larger coupling coefficient at the third cyclotron harmonic, the system becomes vulnerable to parasitic excitation of the $\mathrm{TE}_{-2,4}$ mode at the fundamental cyclotron resonance. Indeed, while the corotating mode $\mathrm{TE}_{2,4}$ has exactly the same dependence of the coupling factor on the beam radius as the $\mathrm{TE}_{0,4}$ mode, the counter-rotating mode $\mathrm{TE}_{-2,4}$ has maximum of the coupling coefficient at the position of the highcurrent outer beam. Since the cutoff frequency of the $\mathrm{TE}_{2,4}$ mode is 0.988 of the one of the $\mathrm{TE}_{0,4}$ mode, the desired operation can be easily suppressed by the $\mathrm{TE}_{-2,4}$ mode excited in the regime of BWO (Fig. 2b). Thus, it is sensible to choose a larger outer beam radius of $0.82 \mathrm{~mm}$, so as to weaken the coupling factor of the parasitic mode (Fig. 2c).

a)

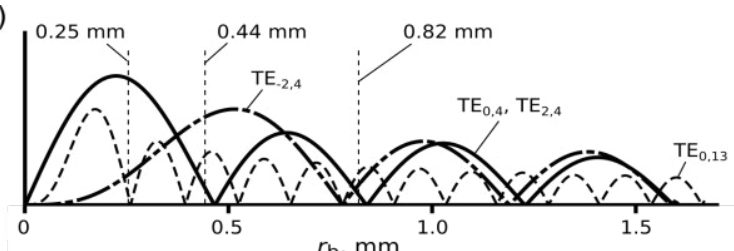

b)

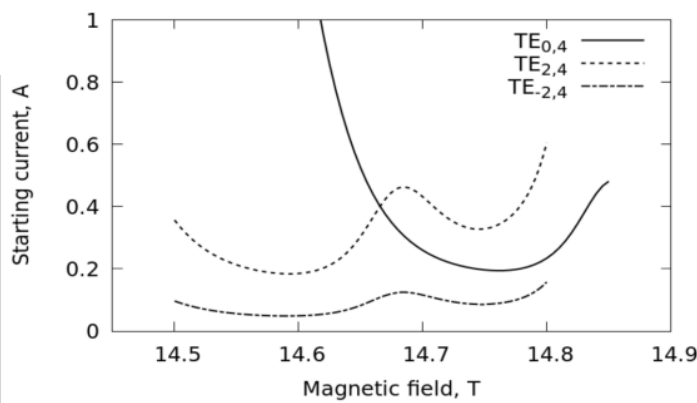

c)

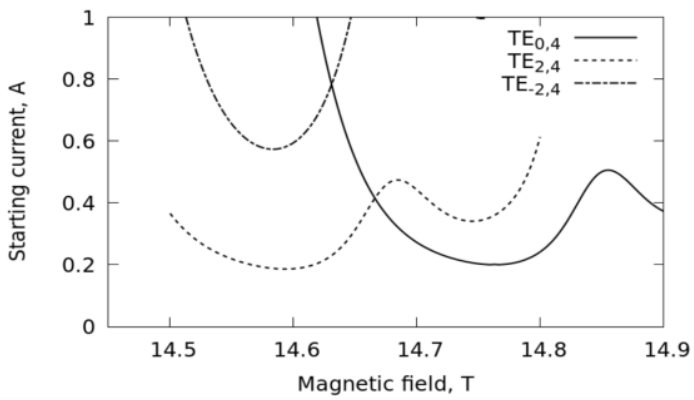

Fig. 2. Dependence of the coupling factors of operating and spurious modes on the beam radius as well as transverse positions of the inner $(0.25 \mathrm{~mm})$ and outer $(0.44 \mathrm{~mm}$ or $0.82 \mathrm{~mm}$ ) beams (a), and starting currents of desired and parasite excitations for the outer beam radii of $0.44 \mathrm{~mm}(\mathrm{~b})$ and $0.82 \mathrm{~mm}$ (c).

\section{Numerical simulations}

In the simulations, both the beams were supposed to have the same electron energy of $22.8 \mathrm{keV}$, the pitch factor of 1.3 , and the $20 \%$ spread in transverse electron velocities. The total operating current was equal to $1 \mathrm{~A}, 0.1 \mathrm{~A}$ being put in the inner electron beam and $0.9 \mathrm{~A}$ in the outer one. For the radii of first and second cavity sections equal to $1.6 \mathrm{~mm}$ and $1.665 \mathrm{~mm}$ correspondingly, the maximum output power at the third cyclotron harmonic at the frequency of $1.2 \mathrm{THz}$ amounted to approximately $6 \mathrm{~W}$ (Fig. 3).
Fig. 4 shows the distribution of operating fields and rf-currents along the cavity in this case.

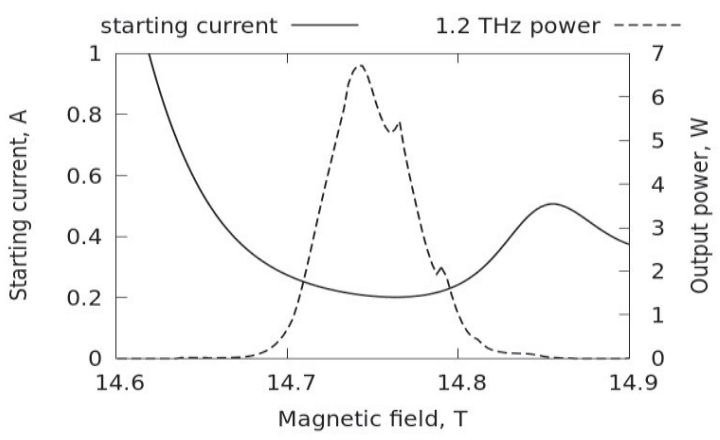

Fig. 3. Dependence of the third cyclotron harmonic output power on the magnetic field for the $1 \mathrm{~A}$ total beam current (simulations).

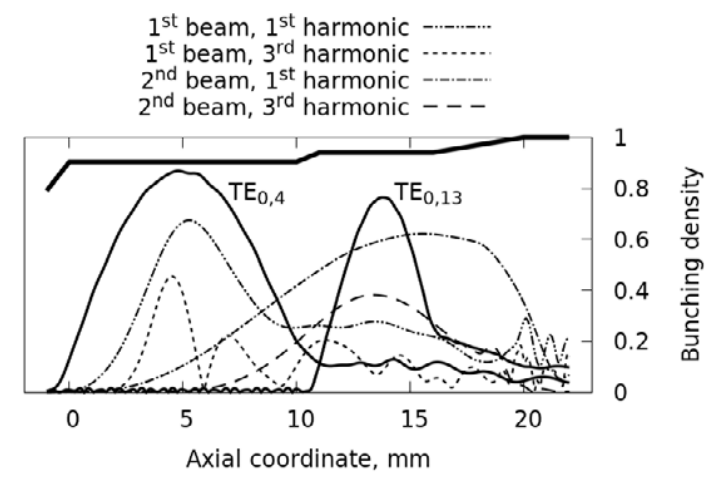

Fig. 4. Distributions of the high-frequency currents along the sectioned cavity for the case of maximum output power.

\section{Conclusion}

A new scheme of a two-resonator frequency gyromultiplier based on the use of two coaxial hollow electron beams is proposed and theoretically investigated. Efficient generation in such a scheme is achieved due to a special choice of the beam radii, when one of the beams provides excitation of the first cavity at the fundamental cyclotron resonance, while the other excites the second cavity at a high cyclotron harmonic. For such a gyromultiplier, the calculations predict the possibility of terahertz generation at the third cyclotron harmonic with a watt-level power.

The work is supported by the Russian Science Foundation, Project No. 14-12-00887.

\section{References}

1. Bandurkin, I.V., Bratman, V.L., Savilov, A.V. Frequency multiplication in gyrotron autooscillators // Tech. Phys. Lett. 2006. V. 32, No. 1. P. 84-87.

2. Savilov, A.V., Nusinovich, G.S. On the theory of frequency-quadrupling gyroklystrons // Phys. Plasm. 2007. V. 14, No. 5, P. 053113.

3. I.V. Bandurkin, V.L. Bratman, G.G. Denisov, Yu.K. Kalynov, A.V Savilov, A.W. Cross, W. He, K. Ronald, A.D.R. Phelps. Single-cavity gyromultipliers // Terahertz science and technology. 2008. V. 1, No. 3, P. 169.

4. I.V. Bandurkin, V.L. Bratman, A.V. Savilov, S.V. Samsonov, A.B. Volkov. Experimental study of a fourthharmonic gyromultiplier. Phys. Plasmas. 2009. V. 16, No. 7, P. 070701. 